\title{
A Novel Engineering Education Approach for Training Outstanding Engineer
}

\author{
Jianhua Deng ${ }^{1, a^{*},}$ Yongbin $\mathrm{Yu}^{2, \mathrm{~b}}$ and Jingye $\mathrm{Cai}^{3, \mathrm{c}}$ \\ ${ }^{123}$ School of Information and Software Engineering of University of Electronic Science and \\ Technology of China, Sichuan Province, P.R.C \\ abc\{jianhua.deng, ybyu, jycai\}@uestc.edu.cn
}

\begin{abstract}
Keywords: Engineering Education; Novel Approach; Student's Abilities;
\end{abstract}
\begin{abstract}
Engineers are trained in the traditional way that cannot provide supply engineers to satisfy the requirements in quality and quantity of industry. Therefore, a program called OEEP is proposed by the ministry education of China in order to cultivate outstanding engineers. In this paper, we proposed a novel engineering education approach called VTI to improve the engineering education in order to cultivate engineer. The industry will deeply participate in education and the students have to spend more than one year in enterprise in order to develop their abilities by multiple ways, such as visiting and training in enterprise, even doing internship.
\end{abstract}

\section{Introduction}

For the past 30 years, as the economy of China always keeps a surprising annual growth rate, China has become the second largest economy in the world. One of the main reasons is that China has the world's largest industrialized and manufacturing nations [1], which means that a large number of engineering talents are demanded. The cultivation of outstanding engineering talents is bound ed to rely on high-quality engineering education. Therefore, strengthening and emphasis on higher engineering education is of great important to promote science and technology development, economy growth, social progress and national security.

This demand is rapidly increasing as the economy is still growing fast. However, it has not produced the supply and quality of engineers needed to satisfy the growing demand, as they are trained in traditional method. Since the engineering field does not distinguish well among different development roles, such as software engineer. Education for software engineers is confounded with education for programmers and other non-engineers. In the next decade, engineering education should prepare students differently for different roles, infuse a stronger engineering attitude in curricula, help students to face the rapid change, and establish credentials that accurately reflect capability. It is a big challenge that how to satisfy the engineering education demand for the higher education. Moreover, traditional education method scants provision for helping students to update their knowledge.

In China, the reform of higher education is being carried out to overcome this challenge and there is a program was proposed by the Ministry Education of P.R.C called Outstanding Engineer Education Program (OEEP). OEEP aims to cultivate a large number of engineers who have innovative capability and other capabilities to satisfy the demand of economy and society. This program also services for the country to achieve the strategy goal of new industrialization road and to help building an innovative country [2]. The OEEP was started on October, 2010 and covered over 340 universities or enterprises in China, currently. Therefore, some new engineering education models are proposed in order to achieve the goal of OEEP.

In this paper, a new engineering education model called VTI is proposed in order to help students to develop skills. Using the VTI model, all students has a lot of opportunities to talk and work with enterprise staffs face to face, do some projects in enterprise and may own some internship job during the 4 years in college. We will propose this model in section III. 
The next of this paper is organized as follows. Some related works will be introduced in section II and, some conclusions will be given in section IV.

\section{Current Engineering Education Model}

In [3], authors proposed a mixed-mode approach, where students are required to do projects with some traditionally taught courses in early years and do some complexity program in later years. It has been demonstrated that the engineering profession and academics are more familiar with the concepts of projects in their professional practice, than with the concepts of problem-based learning.

In [4], an engineering education system called CDIO was proposed which replaces for Conceive - Design - Implement - Operate. They pointed out that the purpose of engineering education is to provide the learning required by students to become successful engineers - technical expertise, social awareness, and a bias toward innovation. Therefore, any approaches to improve engineering education must address two questions: (1) What is the full set of knowledge, skills, and attitudes that engineering students should process as they leave the university, and at what level of proficiency? (2). Is it possible that we can do better at ensuring that students learn these skills?

The ideal of CDIO is based upon the life cycle of software. The reason is that the proposed CDIO collaborators recognize that an engineering education is acquired over a long period and in a variety of institutions, and that educators in all parts of this spectrum can learn from practice elsewhere. The students should have three engineering abilities to satisfy the requirement of the CDIO. First of all, the students should have an abilities to master the engineering knowledge of technical fundamentals; secondly, the ability of leading in the creation of new products, processes and systems is required; finally, students can understand the importance and strategic impact of research and technological development on society.

As the effect of the CDIO, some engineering education methods are proposed based on it. Authors in [6] defined some technical skills that a software engineer should possess, such as the theoretical foundations of the discipline, the design methods of the discipline, the technology and tools of the discipline. It is also found that a gap between learning by studying and learning by doing of software engineering is bigger than that of other engineering. The context of the software engineering should be included the mathematical background, the core of computer science and the essentials of the real world. An approach to improve the vocational education based upon the CDIO engineering education theory is proposed in [5]. The authors also pointed out the disadvantage of vocational engineering education and explain the reason of that.

In this paper, as introduced in section I, we will introduce an engineering education model in order to satisfy the requirement from industry of software engineer.

\section{Proposed Engineering Education Model}

The disadvantage of China's engineering education or software engineering education are embodied in inadequate investment, old conception and lacking practice. Especially, some students don't have a job after graduated as they do not have any engineering experiences. Therefore, a new model is proposed to improve the students' capabilities by some ways, which is called VTI (Visiting - Training - Internship). The VTI is supported by the OEEP and the Visiting - Training - Internship is a system to achieve the goal of the engineering education. The students are required to go into enterprise to finish some work as different roles in different stages during 4 years college.

\section{A. Visiting}

This activity usually is hold in the first year. At that time, the students are fresh and limberness. It means that it is very easy to arouse interests with engineering and to build strong faith of software. However, they lacks skill and knowledge of engineering. Thus, the students will be organized to visit multiple enterprises. They 
have opportunity to talk with the staffs by face to face, which can help them increase knowledge and to open mind. They can touch corporate culture, enhance awareness of requirement of software engineers and professional employment prospects, arouse interests in studying and professional direction, improve motivation of learning as an engineer, and also can guide students' personal future planning and development.

In UESTC (University of Electronic Science and Technology of China), all software engineering students will be organized to visit more than 4 enterprises. They will required to spend 5 days to obverse every enterprise. During that time, student can find some basic rules of an engineer and can experience working atmosphere.

Visiting enterprise is the first step of VTI system, where the goal is to help students to understand the basic requirement as a software engineer in order to arouse strongly motivation of learning. This can help students to think about future planning and push them spend more time and energy in developing engineering skills.

Actually, after back to college, UESTC also invites some enterprises for opening cooperation class, where some engineers with rich engineering experience teach some courses. Usually, the course is about business management, latest technology, and popular tools in industry and so on. This can always make the students full of energy and push them to develop themselves.

\section{B. Training}

The next step of VTI is training which usually happen in the summer of second year. In this stage, students are required to accept a 30 days practical training in enterprise. At first, college collects training position from the cooperation enterprises and publishes to all students. Then students apply the position. The company makes interview and picks someone to own the position. A teacher is assigned to a company in order to help them to manage these students and as a bridge between enterprise, student and college.

During the 30 days, the role of student is quasi formal staff. They have most of the authorization and duty as a formal staff, which means that student can deeply understand corporate culture and know the daily life as a software engineer. The enterprise is authorized to evaluate the training performance of student. The students are required to receive another evaluation from college after back to college. Training provide an opportunity for students to transform the theoretical knowledge to practice, to help them improve the ability of social adaptation and the students' engineering practice ability. This may push students hard working to solve problem which is from practical training.

Then, in the next year, some engineers are invited to give some courses about the skills and tool used in industry in order to help students to handle the development of industry technology. The students are encouraged to join research lab for studying latest technology.

\section{Internship}

The third step of VTI approach is internship, which is organized on the third year. All students are required to work in a company at least for 6 months as an engineer. It means that students will have rich experience as a software engineer in some technology team. They will know how a software program is developed, how to use the knowledge which is learned from college to resolve problem, how to lead a team and how to communicate with customs.

The goal of this stage is (1) to strengthen students' practical ability, such as the skill ofdeveloping software, (2) to really understand the requirement of industry, (3) to fit with the working environment, (4) to introduce enterprise to cooperate with university to educate student, (5) to ready for the job after graduated by reducing the gap between a student in college and a staff in industry.

In a company, one student is in a technology group and is assigned to finish some work. Therefore, they have to improve the practical skills and can accumulate rich experience in engineering which is difficult provided in college. By this way, students can really understand what the requirement is from society and what skills they need to develop. During that time, they have to cooperate with others when they try to finish a work. 
Thus, they have to develop some skills to fit with working environment and improve the society skill to communicate with around persons. All of this will significantly reduce the gap between the ability of students owned and the requirement of industry, which can help students to short the adaptation period into the workplace.

\section{Effect}

This approach has been carried out four years in UESTC and it has the first graduates at the middle of 2015. The effect of VTI approach has been demonstrated. More than $98 \%$ students has a job position before they graduate and the average salary of them is higher than others $18 \%$ in the same university. The human resource of enterprise gives a high evaluation as the students have good skills in engineering and communication.

\section{Summary}

In this paper, we proposed a new engineering education approach called VTI which includes three stages, visiting in the first year to arouse motivation and interests, practical training allows the students to have a short internship for feeling works of engineering and internship is the third stage where they can rapidly develop engineering skills to meet the requirement from industry. Until now, the results demonstrated that that this approach can significantly improve quality of graduates.

\section{References}

[1] W. Zhou, "Engineering education reform needs "Foundation"," Shanghai Education, 2007, 108, pp. 18-20.

[2] http:/www.moe.gov.cn/publicfiles/business/htmlfiles/moe/s3860/201102/115066.html.

[3] Mills, Julie E., and David F. Treagust. "Engineering education-Is problem-based or project-based learning the answer?." Australasian Journal of Engineering Education 3.2 (2003): pp. 2-16.

[4] E. F. Crauley, S. Ostulnd, J. Malmqvist, D.R. Brodeur, "Rethinking Engineering Education - the CDIO Approach,” Spring, September, 2007, pp. 10.

[5] Q. Xu, D. Xie, "VocationalEngineering Education Model Research," the 7th International Conference on Computer Science \& Education(ISSCE 2012), pp. 1457 - 1459, July 14 - 17, 2012, Melbourne, Australia.

[6] C. Ghezzi, D. Mandrioli, "The Challenges of Software Education," Proceedings of the 27th international conference on Software engineering (ICSE '05), pp. 637 - 638, 15 - 21 May, 2005, Missouri, USA. 